\title{
Sustentabilidade de agroecossistemas familiares de cultivo de manga irrigada versus sequeiro: aplicação do MESMIS
}

Esta pesquisa teve como objetivo avaliar a sustentabilidade em agroecossistemas de cultivo da manga irrigada versus sequeiro, mediante aplicação dos 4 passos do método MESMIS em práticas nos municípios da zona rural das cidades de Acari e Cruzeta, localizadas na região semiárida do Rio Grande do Norte, dada a sua relevância em ajudar na avaliação da sustentabilidade dos sistemas de manejo dos agroecossistemas, com ênfase no contexto dos agricultores rurais. A coleta de dados ocorreu por meio da pesquisa de campo, com visitas e observação direta, com participação dos agricultores locais. 0 método utilizado abordou uma série de indicadores ambientais, sociais e econômicos para diagnosticar a sustentabilidade em sistemas agrícolas. A partir dele, foi possível identificar as potencialidades oriundas do MESMIS para maximizar o desenvolvimento sustentável dos agroecossistemas que, devido às suas características de adaptabilidade às realidades locais e ao seu uso em diferentes tipos de análises, permitiu a identificação de novos objetivos para a sua utilização.

Palavras-chave: Agricultura familiar; Indicadores; Método de avaliação; Sustentabilidade.

\section{Sustainability of family agroecosystems of irrigated mango crop versus rainfed: application of MESMIS}

\begin{abstract}
The objective of this research was to evaluate the sustainability in irrigated mango versus rainfed agroecosystems by applying the 4 steps of the MESMIS method in practices in the rural municipalities of the cities of Acari and Cruzeta, located in the semi-arid region of Rio Grande do Norte, Brazil. given its relevance in helping to assess the sustainability of agro-ecosystem management systems, with an emphasis on the context of rural farmers. Data collection occurred through field research, with visits and direct observation, with the participation of local farmers. The method used addressed a series of environmental, social and economic indicators to diagnose sustainability in agricultural systems. From it, it was possible to identify the potentialities of MESMIS to maximize the sustainable development of agroecosystems that, due to their adaptability characteristics to local realities and their use in different types of analysis, allowed the identification of new objectives for their use.
\end{abstract}

Keywords: Family farming; Indicators; Evaluation method; Sustainability.

Topic: Desenvolvimento, Sustentabilidade e Meio Ambiente

Reviewed anonymously in the process of blind peer.
Received: 17/06/2019

Approved: 17/07/2019

Houtran Lima da Silva (iD)

Instituto Federal do Rio Grande do Norte, Brasil

http://lattes.cnpq.br/6191536800052473

http://orcid.org/0000-0003-1172-4525

houtran.silva@gmail.com

Gerda Lúcia Pinheiro Camelo (iD)

Instituto Federal do Rio Grande do Norte, Brasil

http://lattes.cnpq.br/6200316595995035

http://orcid.org/0000-0002-3986-408X

gerda.cameloo@ifrn.edu.br

Referencing this:

SILVA, H. L.; CAMELO, G. L. P.. Sustentabilidade de agroecossistemas familiares de cultivo de manga irrigada versus sequeiro: aplicação do MESMIS. Revista Ibero-Americana de Ciências Ambientais, v.10, n.4, p.191-208, 2019. DOI: http://doi.org/10.6008/CBPC2179$\underline{6858.2019 .004 .0015}$ 


\section{INTRODUÇÃO}

Historicamente, a agricultura familiar tem sido o modelo operacional mais comum, de modo a garantir o crescimento do setor agrícola durante séculos. Desse modo, é notório que essa modalidade, no Brasil, possui uma diversidade agrícola próspera, com capacidade de sobrevivência e adaptação às novas situações que ocorrem constantemente na sociedade.

Contudo, apesar desses benefícios, dispõe de infinitos complicadores para a sua apreciação, como o tamanho das propriedades e seu entrelaçamento com a agricultura de subsistência, que de acordo com as diretrizes para a formulação da Política Nacional da Agricultura Familiar e Empreendimentos Familiares Rurais, estabelecido na Lei no 11.326, de 24 de julho de 2006, onde no artigo 3ำ, são destacadas como características primordiais para se enquadrar como agricultores familiares: não deter área maior do que quatro módulos fiscais; utilizar mão de obra familiar como principal atividade econômica; obter o percentual mínimo de renda das atividades econômicas a partir do seu estabelecimento; e dirigir o seu estabelecimento com a família (BRASIL, 2006).

Sabe-se que a população mundial está em constante crescimento e a progressiva demanda por alimentos tem provocado uma tendência ao aumento do consumo, ao passo que a estratégia ganha novas proporções frente ao rendimento de produção agrícola e o aumento de preços. A agricultura familiar proporciona, portanto, ambiente favorável ao fortalecimento do setor agrícola, considerando a inclusão de famílias que vivem no campo, as quais, de acordo com Buainain (2006), exploram minifúndios, ou seja, apropriam-se de pequena extensão em condições de extrema pobreza, a exemplo de produtores inseridos no moderno agronegócio que gera renda superior, variadas vezes, a que define a linha da pobreza.

O final da década de 1960, no Brasil, foi marcado por um modelo de extensão rural que foi difundido e preconizado em meio à Revolução Verde e/ou modernização da agricultura, a qual tinha como objetivo principal o difusionismo. Esse modelo de extensão rural não considerava os saberes tradicionais dos agricultores, desprezando, assim, as condições sociais, culturais, econômicas e políticas em disputa (OLIVEIRA, 2015). Para Castro (2015), entre esses princípios, incluía a inclusão da inovação tecnológica no âmbito das atividades agropecuárias e a difusão da inovação nessas atividades.

É possível notar, nas contribuições de Delgado et al. (2017), que até a década de 1990 a agricultura familiar brasileira não havia conhecido um conjunto tão amplo de instrumentos de políticas públicas voltadas à proteção e ao desenvolvimento desta categoria social. É no decreto de no 1946 de 28 de julho de 1996 que foi criado o Programa Nacional de Fortalecimento da Agricultura Familiar (PRONAF), considerado um marco da criação de políticas públicas diferenciadas, delineando o reconhecimento do Estado brasileiro frente à importância econômica e social da agricultura familiar, que para Schneider et al. (2004) o programa tinha por finalidade prover crédito agrícola, bem como apoio institucional aos pequenos produtores rurais que vinham sendo excluídos das políticas públicas até então existentes e que encontravam uma série de dificuldades em se manter no campo. 
De acordo com Vargas et al. (2012), a forma de desenvolvimento baseado na sustentabilidade passa a ser, a datar dos anos 90, uma nova necessidade, onde aumentaram as pesquisas e a defesa da agricultura familiar como alternativa para o desenvolvimento rural sustentável. De tal modo, é nesse enviés que, as políticas públicas voltadas para a agricultura introduziram uma nova categoria política, chamada de: agricultura familiar, da qual, esta iniciativa procurava implantar nas políticas públicas um conjunto vasto para os agricultores rurais, que até então, eram afastados desses programas governamentais.

De acordo com o Ministério do Desenvolvimento Agrário (BRASIL, 2016), a agricultura familiar baseada na gestão da propriedade é compartilhada pela família e a atividade produtiva agropecuária, é uma junção dos substantivos agricultura e pecuária, das quais são as principais fontes geradora de renda. E desse modo, que na Assembleia Geral de 2015 da Organização das Nações Unidas (ONU), uma das expectativas da agenda 2030 é que a agricultura sustentável dobre a produtividade agrícola e a renda dos pequenos produtores de alimentos, a fim de garantir sistemas sustentáveis de produção e o fortalecimento da capacidade de adaptação às mudanças do clima, às inundações e a outros desastres.

Petersen et al. (2017), afirma que a produção econômica gerada no agroecossistema ${ }^{1}$ contribui para a economia no âmbito da comunidade, uma vez que, parte importante da renda produzida é canalizada para remunerar agentes econômicos sejam, produtores e fornecedores de insumos etc. Assim, o agroecossistema contribui para a geração de trabalho e renda dos produtores rurais, para uma melhor qualidade de vida na propriedade, possibilitando a manutenção da família no campo, oferecendo condições dignas, segurança alimentar com produção de alimentos de alta qualidade, identidade e sociabilidade rural, bem como, preserva os recursos naturais e sua paisagem rural.

Com os problemas relacionados à seca que tem comprometido o desenvolvimento e o crescimento das atividades produtivas no campo e tem ocasionado uma redução na biodiversidade local, alguns agricultores assumem que a fruticultura irrigada e de sequeiro ${ }^{2}$ promoveram contribuições, apesar das dificuldades adversas e das outras de situações. Em 2013, segundo a Empresa Brasileira de Pesquisa Agropecuária (EMBRAPA), a agricultura irrigada apresenta-se como a alternativa mais coerente diante das incertezas do risco climático, e o cultivo de sequeiro pode ser viabilizado com espécies tolerantes à seca e de ciclo curto.

A fruticultura irrigada, importante segmento do setor agrícola, brota como uma atividade dinâmica, ou seja, com ação, produzindo alimentos de maior valor agregado, com um processo de produção agrícola e/ou seja, avançado, baseado em tecnologias modernas capazes de tornar a atividade mais competitiva. Já as espécies frutíferas nativas ou aquelas adaptadas às condições de sequeiro, contribuem para o desenvolvimento de uma fruticultura competitiva, podendo ser, diversificada, com potencial econômico para agricultura familiar e que contribui com a renda dos agricultores familiar que dependem da agricultura de

\footnotetext{
${ }^{1}$ De acordo com Petersen et al. (2017), entende-se como uma unidade social de apropriação e conversão de bens ecológicos em bens econômicos, manejado de forma a aumentar a produtividade seja de um grupo seleto de produtores, como consumidores.

${ }^{2}$ A agricultura de sequeiro é o cultivo sem irrigação em regiões onde a precipitação anual é inferior a 500mm (QUARANTA, 2000).
} 
sequeiro, pois, com os efeitos das imprevisibilidades no regime das chuvas o sistema de cultura de sequeiro é geralmente um fator limitante.

Destaca-se que na região Seridó do estado do Rio Grande do Norte (RN) que até então era dominada pela pecuária no final do século XIX, popularizou-se pelo plantio de algodão. Completando, Silva et al. (2017), esta região tem um histórico de ocupação do espaço em que as atividades econômicas exerceram ao longo do tempo uma forte pressão sobre os recursos naturais, e hoje os agricultores produzem culturas permanentes como a manga para complementar a dieta alimentar durante boa parte do ano.

A manga é uma espécie frutífera de clima tropical, cultivada em quase todos os estados brasileiros. No cenário nacional, a Região Nordeste é considerada a grande produtora de manga para exportação e seus sistemas de cultivo são os mais tecnificados no país, em função de suas excelentes condições para o seu desenvolvimento e produção, nos principais polos de irrigação da região semiárida (COSTA et al., 2008).

Embora exista número significativo de cultivares de manga no Brasil, aspectos como preferência de consumidores, produtividade, tolerância a pragas e doenças, qualidade do fruto, resistência à seca, porte e resistência ao frio são responsáveis pela pouca quantidade de cultivares com igual potencial produtivo e de qualidade em plantios comerciais para consumo in natura. Em termos botânicos de classificação hierárquica, as mangueiras produtoras de frutos comestíveis nestas duas comunidades rurais é a Tommy Atkins que são as plantas da classe das Magnoliopsida, ordem Sapindales, família Anacardiaceae, gênero Mangifera e Espécie Mangifera indica (BRASIL, 2007).

Tendo em vista a importância desse cultivo para as cidades e regiões do Seridó, bem como a atuação que a agricultura familiar possui atualmente nesta atividade, o presente artigo se propõe avaliar a sustentabilidade de agroecossistemas familiares, comparando o cultivo da manga irrigada versus sequeiro na zona rural do município de Acari e Cruzeta (RN), mediante aplicação de um método que foi criado em 1995 por um grupo interdisciplinar e multi-institucional do México (MASERA et al., 2000).

Tudo isso com o intuito de traduzir princípios gerais de sustentabilidade em definições operacionais, indicadores e práticas no contexto da gestão de recursos naturais em comunidades camponesas, chamado: Marco para Avaliação de Sustentabilidade de Recursos Naturais Incorporando Indicadores de Sustentabilidade (MESMIS), que propõe linhas de trabalho para os pontos críticos, almejando alcançar maior sustentabilidade do sistema com foco na implementação de estratégias e elementos de análise em processo de melhoria contínua, percorrendo os 4 (quatros) primeiros passos do ciclo avaliativo do referido método.

Por essa razão, esta pesquisa foi realizada em duas comunidades da região Seridó, situados na zona rural das cidades de Acari e Cruzeta (RN), escolhidas por serem, respectivamente, responsáveis pela produção e comercialização da manga e pelo domicílio dos agricultores, pois nelas, além de haver predominância de agroecossistemas familiares, existe à aproximação dos atores sociais envolvidos nos vários processos pertinentes ao cultivo da manga irrigada e de sequeiro. O método foi escolhido por ser aplicável em propriedades agrícolas produtivos, por ser flexível em relação a sua estrutura, de forma a estimular a percepção de cada indivíduo no que diz respeito ao seu ambiente produtivo, da mesma maneira, que se 
adapta e/ou adequa as necessidades específicas dos agroecossistemas e por promover maior interação com os agentes participantes e permitindo assim a comparação da sustentabilidade entre os agroecossistemas.

Neste artigo, os objetivos visam promover ações voltadas à preservação e à sustentabilidade dos recursos naturais, propondo linhas de trabalho para os pontos críticos e almejando alcançar maior sustentabilidade do sistema, com foco na implementação de estratégias e elementos de análise como processo de melhoria contínua.

\section{REVISÃO TEÓRICA}

\section{Concepções teóricas norteadoras da investigação: agricultura no contexto da sustentabilidade}

A diversificação e a multifuncionalidade da agricultura familiar são discussões que vêm ganhando intensidade conduzida pelo desenvolvimento sustentável. Seu processo produtivo é realizado pelos próprios agricultores, os quais trabalham com a diversificação, utilizando o trabalho familiar, a geração de emprego e renda, o que revela que a sua contribuição supera o conceito de que a agricultura familiar é um lugar de atraso ou simplesmente de retrocesso ao desenvolvimento.

Desse modo, incentivar a agricultura familiar é, também, manter a sustentabilidade da economia em grande parte no Brasil, uma vez que essa atividade tem, atualmente, uma grande influência na economia de muitas regiões, pois ela está mais presente nas mesas do brasileiro do que se imagina (DEIMLING et al. 2015). Embasado nessa perspectiva, surgem estudos envolvendo a avaliação da sustentabilidade na agricultura, de forma a identificar dificuldades de diagnóstico pela abordagem reducionista que ainda prevalece entre profissionais das ciências agrárias e entre muitos outros pesquisadores, o que gera dificuldades para entender a complexidade do tema, o qual requer uma abordagem holística e sistêmica (SARANDÓN, 2002).

$\mathrm{Na}$ análise da sustentabilidade ${ }^{3}$ de agroecossistemas ${ }^{4}$ vários temas tem sido abordados referentes à produção agrícola de base ecológica como à dinâmica socioambiental, entretanto, é necessário que se encontre formas de operacionalizar, buscando a autonomia ou o empoderamento das comunidades rurais para que possam desempenhar soluções de seus problemas, pois, o monitoramento da sustentabilidade de seus agroecossistemas é sentida pelos agricultores familiares de modo a tornar mais efetiva à necessidade de tomada de decisões.

De acordo com a EMBRAPA, no Brasil, a agricultura familiar compreende aproximadamente 4,3 milhões de unidades produtivas (cerca de $84 \%$ do total) com a média de 14 milhões de pessoas ocupadas, representando cerca de $74 \%$ do total das ocupações distribuídas em $25 \%$ da área total. Percebe-se, assim, que a agricultura permite manter outras atividades que não necessariamente estão diretamente ligadas a ela, como o pequeno comércio e os serviços públicos, logo, a agricultura é um setor chave no desenvolvimento rural. Na concepção de Martins (2018), uma agricultura sustentável deve ser a que oferece uma renda viável para agricultores ao longo de toda a cadeia comercial em qualquer país, além de fornecer

\footnotetext{
${ }^{3}$ Para Sarandón (2002), a sustentabilidade é um conceito embaraçado em si porque pretende desempenhar ao mesmo tempo vários objetivos que envolvem dimensões produtivas, ecológicas ou ambientais, sociais, culturais, econômicas e, fundamentalmente, temporais.

${ }^{4}$ Esse conceito proporciona uma estrutura com a qual podemos analisar os sistemas de produção de alimentos como um todo, incluindo seus conjuntos complexos de insumos e produção e as interconexões presentes no território" (CÂNDIDO et al., 2015).
} 
alimentos nutritivos e adaptarem-se às mudanças climáticas, a fim de reduzir os gases de efeito estufa e o desmatamento.

De acordo com o Censo Agropecuário de 2017 do Instituto Brasileiro de Geografia e Estatística (IBGE), referente à agricultura familiar, o estado do Rio Grande do Norte possui em torno de 63.000 (sessenta e três mil) estabelecimentos agrícolas e/ou agricultores, ao passo que, em 2006, esse número chegou a 83.000 (oitenta e três mil). Assim sendo, percebe-se que houve uma diminuição em torno de $23,64 \%$ na área de estabelecimentos agrícolas, ressaltando, assim, a menor concentração de terras entre os grandes e médios estabelecimentos, quando comparado ao censo vigente.

Nesta continuidade, e com propósito de corroborar o segmento da agricultura familiar no Brasil, foram elaborados, criados e implantados alguns programas que fazem parte das políticas públicas, tais como: Programa Nacional de Fortalecimento da Agricultura Familiar (PRONAF) em 1995; Programa de Aquisição de Alimentos (PAA) em 2003; Programa Nacional de Alimentação Escolar (PNAE) em 2009 aliados à estratégia intersetorial $^{5}$ de combate à fome e à pobreza por meio do Programa Fome Zero (PFZ) e das ações do Plano Brasil Sem Miséria (CUNHA et al., 2017). Estes programas, são conceituados de forma característica como multifuncionalidade da agricultura familiar. Percebe-se que o problema da fome é sobretudo político, que merece análise multifacetada, além de um olhar estrito de fomento à produção de alimentos.

Como assinala Brose (1999), as vantagens apresentadas pela agricultura familiar são maiores no que diz respeito à estabilidade e capacidade de adaptação, devido à sua ênfase na diversificação e estratégia de desenvolvimento e a maior capacidade de adaptação de seu processo decisório. E para que não haja um monopólio e intermediários que se apropriem do valor agregado da produção, não podem deixar à sua própria sorte frente às forças do mercado.

Mascarenhas (2004), a agricultura sustentável busca abarcar todos os sistemas nos quais haja uma clara inversão do sentido ecológico alinhado à visão alternativa de sustentabilidade da agricultura convencional. Dessa forma, pode significar, ainda, um caminho para garantir a segurança alimentar de uma parcela considerável da sociedade, promovendo uma alternativa ao progresso e ao bem-estar no meio rural para garantir melhores condições de vida à população.

Assim sendo, para mensurar a agricultura sustentável, é necessário utilizar indicadores de sustentabilidade para auxiliar no acompanhamento da operacionalização do desenvolvimento, pois indicadores surgem de valores e geram, também, valores, além de apontar os elementos que favorecem a sustentabilidade de uma determinada atividade. Desse modo, permite a análise do agroecossistema e evidencia seu desempenho, eficiência, produtividade e os problemas, a fim de serem encontradas soluções.

\section{Sistemas de Indicadores de Agroecossistemas: como mensurar a sustentabilidade}

A partir da explanação da agricultura no contexto da sustentabilidade, há a necessidade de mensurar o valor de certas grandezas e o grau de sustentabilidade desejado, o que resulta na necessidade de se buscar

\footnotetext{
${ }^{5}$ Para o Ministério do Desenvolvimento Social (MDS), essa estratégia tem como objetivo prevenir e controlar a obesidade na população brasileira através de ações intersetoriais, a fim de, promover a alimentação adequada e saudável e a prática de atividade física no ambiente em que se vive.
} 
indicadores de sustentabilidade nos agroecossistemas. Cândido et al. (2015) evidencia que indicadores de sustentabilidade são parâmetros que podem avaliar o desenvolvimento de uma atividade econômica em termos de sustentabilidade, a partir de valores quantitativos e análise do agroecossistema em termos econômicos, ambientais e sociais. Para avaliar ou mensurar a sustentabilidade através dos indicadores, o agroecossistema deve se encontrar mais adequado aos problemas observados. De acordo com Ferraz et al. (2004), os fatores essenciais que caracterizam uma eficiência dos indicadores frente à unidade de análise observam que

[...] os indicadores de sustentabilidade devem ser capazes de avaliar o comportamento do agroecossistema em relação à produtividade: alto ou baixo rendimento dependendo dos recursos naturais; estabilidade: grau no qual a produtividade ou capacidade produtiva se mantém constante; elasticidade: capacidade de recuperação do sistema frente a fatores externos; e equidade: distribuição equitativa do recurso econômico e dos benefícios/riscos gerados pelo manejo do sistema. Os indicadores devem apresentar, entre outros fatores, simplicidade de mensuração e repetibilidade ao longo do tempo, sensibilidade para detectar mudanças no sistema e permitir o cruzamento com outros indicadores.

Para Sarandón (2002), não existe uma única maneira de versar a avaliação de sustentabilidade, pois depende do objetivo ou do tipo de pergunta que está sendo respondida. Nesse sentido, um indicador de mensuração se define como uma variável selecionada e quantificada que permite o pesquisador observar uma tendência que não é facilmente detectável. A complexidade e a multidimensionalidade da sustentabilidade tornam necessário transformar aspectos complexos em valores claros, objetivos e gerais, os quais são, por sua vez, denominados indicadores.

É nesse cenário, que o método MESMIS permite comparar um manejo de avaliação de sustentabilidade de sistemas de produção agrícolas a fim de gerar um processo cíclico que, integrado efetivamente à avaliação no processo de tomada de decisão, melhora a probabilidade de sucesso no desenho de alternativas e a implementação de projetos de desenvolvimento (MASERA et al., 2000).

De acordo com Masera et al. (2000), o programa MESMIS considera simultaneamente cinco objetivos estratégicos interrelacionados: integra a teoria de sistemas complexos em avaliações de sustentabilidade; desenvolve e atualiza um sistema abrangente e coerente de estrutura de avaliação de sustentabilidade; facilita os processos de aprendizagem pelos quais as partes interessadas podem entender, usar e dar feedback sobre os conceitos, ferramentas e resultados dessas avaliações; valida, promovendo vários estudos de caso; e promove e facilita a participação de todas as partes interessadas na avaliação processo.

O MESMIS, além de permitir comparar um agroecossistema, compreende, de forma integral, as ameaças e as oportunidades para a sustentabilidade dos sistemas de manejo em busca de um desenvolvimento social mais equitativo e ambientalmente saudável das comunidades rurais (MASERA et al., 2000; CAMELO et al., 2013). No entanto, vale ressaltar que, embora esse método seja bastante preciso, seu sucesso advém da escolha dos indicadores corretos.

A utilização do método MESMIS, para Verona (2010), procede como um processo que permite adaptações de acordo com as necessidades específicas dos agroecossistemas que estão sendo mensurados. Sendo assim, a participação dos agricultores da área de estudo é aconselhável para reconhecer e identificar 
os pontos críticos positivos/negativos, seja no levantamento de dados ou nas discussões e entrevistas (MAZERA et al., 2000).

Assim sendo, a mensuração da sustentabilidade busca compreender o contexto e as causas dos resultados identificados de forma que fiquem claros as potencialidades de cada agroecossistema e que se compreendam a situação encontrada no nível de contribuição. Para isso, são necessárias informações que subsidiem ações para melhorias nos agroecossistemas por meio da avaliação do MESMIS, aplicado, em nosso estudo, em unidades de cultivo da manga.

\section{O cultivo da manga irrigada e de sequeiro na comunidade da zona rural das cidades de Acari e Cruzeta- RN: a inserção da agricultura familiar}

Como a manga é uma planta tipicamente tropical, seu bom desenvolvimento exige calor constante, elevada umidade e boa distribuição de chuvas, sem tolerância ao frio excessivo, aos ventos ou às geadas. Por esse motivo, a mangueira é cultivada em altitudes inferior a $600 \mathrm{~m}$, para que não haja influência negativa no ciclo, na produtividade e na qualidade do fruto (SILVA et al., 2002).

Sobre o cultivo da manga (Tommy Atkins) na comunidade rural dos municípios de Acari e Cruzeta, percebe-se que, no intervalo dos últimos 13 anos, a quantidade de manga produzida não apresentou oscilações em relação a sua produtividade (IBGE, 2017). De acordo com o último censo Agropecuário de 2017, promovido pelo IBGE, a zona rural do município de Acari produziu 336 toneladas, ficando em 19 lugar, ao passo que a zona rural do município de Cruzeta produziu 27 toneladas, estando no 79ㅇ lugar. Segundo a EMBRAPA, a agricultura irrigada apresenta-se como a alternativa mais coerente diante das incertezas do risco climático, e o cultivo de sequeiro podem ser viabilizados com espécies tolerantes à seca e de ciclo curto.

$\mathrm{Na}$ área da pesquisa, a manga é cultivada pelos produtores de forma irrigada e de sequeiro, tendo sua produtividade média de 336 t.ha $^{-1}$ no tipo irrigado, na zona rural do município de Acari, e a produtividade média do cultivo de sequeiro da zona rural do município de Cruzeta de 27 t.ha ${ }^{-1}$. Desse modo, o uso da irrigação possibilita o aumento da cultura e otimiza a produção, permitindo o aumento em aproximadamente doze vezes mais quando comparada com o cultivo de sequeiro. Os benefícios da irrigação na cultura da manga são acentuados, pois o efeito potencial da irrigação contribui para o aumento de produtividade e a melhoria da qualidade da fruta. Percebe-se, assim, que há o aumento de produtividade da fruta decorrente da irrigação, mesmo sem monitoramento técnico adequado.

\section{METODOLOGIA}

A pesquisa foi realizada na região noroeste do estado de Rio Grande do Norte, onde foram percorridos no mês de setembro de 2018, visitas a quatro comunidades rurais, sendo duas na zona rural da cidade de Acari e duas na zona rural da cidade de Cruzeta, na região Seridó. Nesta fase realizou-se visita as famílias com o objetivo de conhecer os agroecossistemas, a observação direta dos agricultores locais e a coleta dos dados. Nesse sentido, a área apresenta produção de origem familiar, necessitando assim, a 
aplicação da metodologia MESMIS para que se possa verificar e analisar a sustentabilidade dos agroecossistemas familiar do cultivo de manga irrigado versus de sequeiro.

Optou-se por pesquisar quatros agroecossistemas da região, considerando particularidades entre os quais possuem ênfase na atividade de produção da manga irrigada e de sequeiro, sob o sistema de monocultura. Os critérios para selecionar os quatros agroecossistemas foram: tamanho das propriedades, cultivos de manga irrigada, cultivo da manga sequeiro e os modos de cultivo. Todas as propriedades estavam localizadas na zona rural, conforme a figura 1.

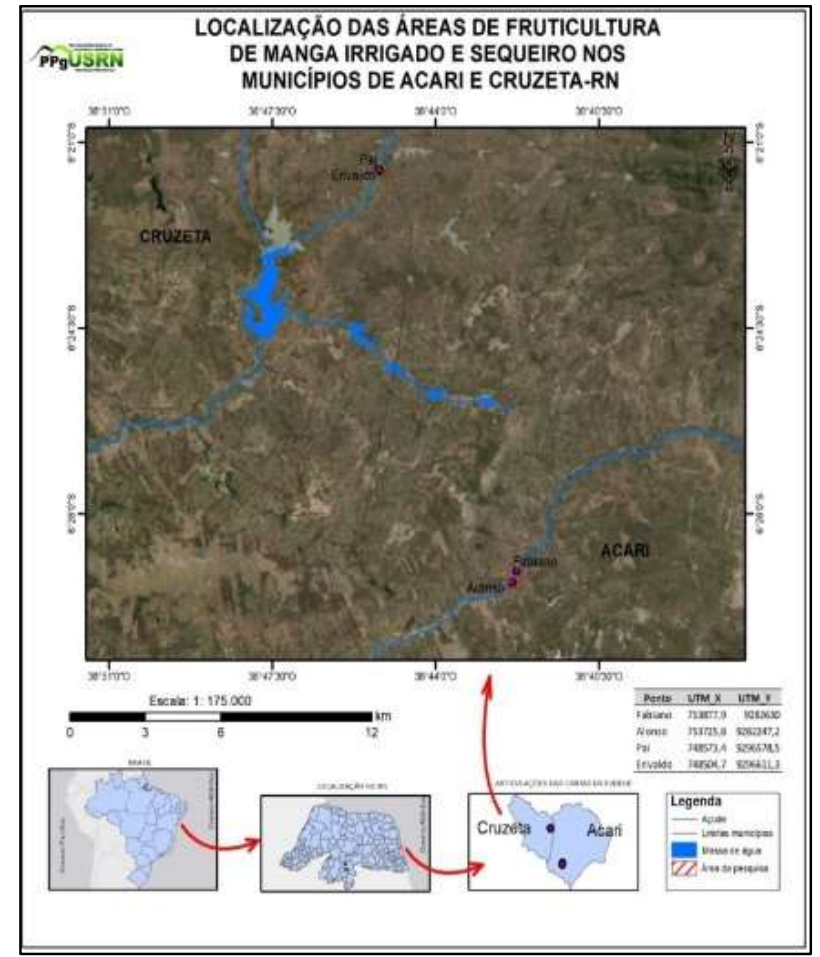

Figura 1: Localização da área objeto de estudo.

O artigo abordará resultados da execução dos passos 1, 2, 3 e 4 do método MESMIS nos quatro agroecossistemas familiares. Estes 4 (quatro) passos são de importância essencial para o ciclo do processo, pois os agroecossistemas avaliados devem ser entendidos de maneira integral, para que possa identificar dos pontos críticos dos sistemas, que dizer, fatores limitantes e possibilidades para a sustentabilidade destes.

Decerto, a escolha de um caminho para as investigações não ocorre aleatoriamente e descrever o tipo de pesquisa e o modo como ela foi aplicada depende, portanto, do objeto de pesquisa, do enfoque ou de qual lugar se deseja abordar. Uma das suas principais tarefas foi obter um conhecimento da literatura, o que o permitiu ter conhecimento do que já havia sido estudado sobre o assunto (FONSECA, 2002; GIL, 2010). Por esse motivo, a presente pesquisa foi iniciada por meio do estudo exploratório e revisão de literatura, buscando familiarizar-se com o fenômeno que foi investigado, de modo que estudos subsequentes possam ser concebidos com uma maior compreensão, entendimento e precisão e que poderá auxiliar no planejamento e no desenvolvimento rural dos agricultores, contribuindo, de alguma forma, com o alcance de uma visão mais holística da complexa realidade do meio rural. 
A pesquisa iniciou pela fase de revisão da literatura, que teve por finalidade a preparação do ponto de vista teórico para identificar as potencialidades e resolver limitações identificadas dos agroecossistemas, podendo auxiliar no planejamento e no desenvolvimento rural dos agricultores, e contribuir de alguma forma para o alcance de uma visão mais holística da complexa realidade do meio rural. Em seguida, a obtenção dos dados primários (obtidos no campo com os quatros chefes de família), secundários (IBGE, 2017), e o ciclo avaliativo proposto por Masera et al. (2000), utilizando os 4 (quatro) passos de aplicação do método MESMIS em torno da amplitude das dimensões propostas, além da observação direta do pesquisador na busca de subsídios que permitirão confrontar esses dados, com a perspectiva de compreender com maior precisão a realidade dos agroecossistemas.

Nesse contexto, a abordagem qualitativa preocupou-se, portanto, com aspectos da realidade que não podem ser quantificados, centrando-se na compreensão e na explicação da dinâmica das relações sociais. Gallopín (1996), cita que é preferível a utilização de indicadores qualitativos em pelo menos três situações diferentes: da indisponibilidade de informações quantitativas; o atributo de interesse não é quantificável; e quando as considerações de custo se tornam determinantes.

A partir dela, pode-se descrever, ainda, a complexidade de determinado problema, analisar a interação de certas variáveis, compreender e classificar processos dinâmicos vivenciados (GERHARDT et al., 2009). Além do mais, podemos citar como por exemplo, a mensuração do rendimento da manga, os dados da sua produção, bem como, a avaliação em relação ao custo/benefício da produção da manga, renda familiar, à disponibilidade e à qualidade da água, solo, entre outros.

A pesquisa está estruturada sob o tipo de pesquisa de campo, que para Marconi et al. (2007), é uma etapa que é realizada após o estudo bibliográfico, com o objetivo de conseguir informações e/ou conhecimentos acerca de um problema, ou, ainda, descobrir novos fenômenos ou as relações entre eles; considerando a caracterização empírica (busca de dados relevantes) dos agroecossistemas familiares. Segundo Gonsalves (2001),

[...] a pesquisa de campo é o tipo de pesquisa que pretende buscar a informação diretamente com a população pesquisada. Ela exige do pesquisador um encontro mais direto. Nesse caso, o pesquisador precisa ir ao espaço onde o fenômeno ocorre, ou ocorreu e reunir um conjunto de informações a serem documentadas [...].

No que diz respeito à pesquisa de campo, é possível dizer que ela contribui com organização das informações no intuito de reunir dados para melhor compreender a realidade investigada, além de desenvolver uma visão contemporânea e eficaz do objeto de estudo, de modo a ir de encontro ao que se pretende pesquisar.

Sendo assim, a pesquisa foi realizada a partir da observação dos procedimentos comuns dos agricultores em relação a seu agroecossistema, com ênfase na descrição dos fatos sobre o cultivo da manga irrigada e de sequeiro com os quatro 'chefes' de família que residem no perímetro destas comunidades, gerando informações quantitativas e qualitativas, a fim de capturar as características de forma transparente e imparcial, bem como caracterizar os agroecossistemas por meio das informações que foram coletadas em campo. 
A fim de contemplar da melhor forma possível o problema de investigação, foi necessário adotar uma metodologia de pesquisa científica que pôde ser classificada e definida conforme sua abordagem, finalidade e procedimentos técnicos empregados, a fim de determinar o método que possibilitará chegar a esse conhecimento, conforme sugere Gil (2010). Assim, foi utilizado o método MESMIS, o qual nos possibilitou chegar a tal conhecimento. Como pode ser observado na figura 2, a metodologia do MESMIS é composta por um ciclo de seis passos.

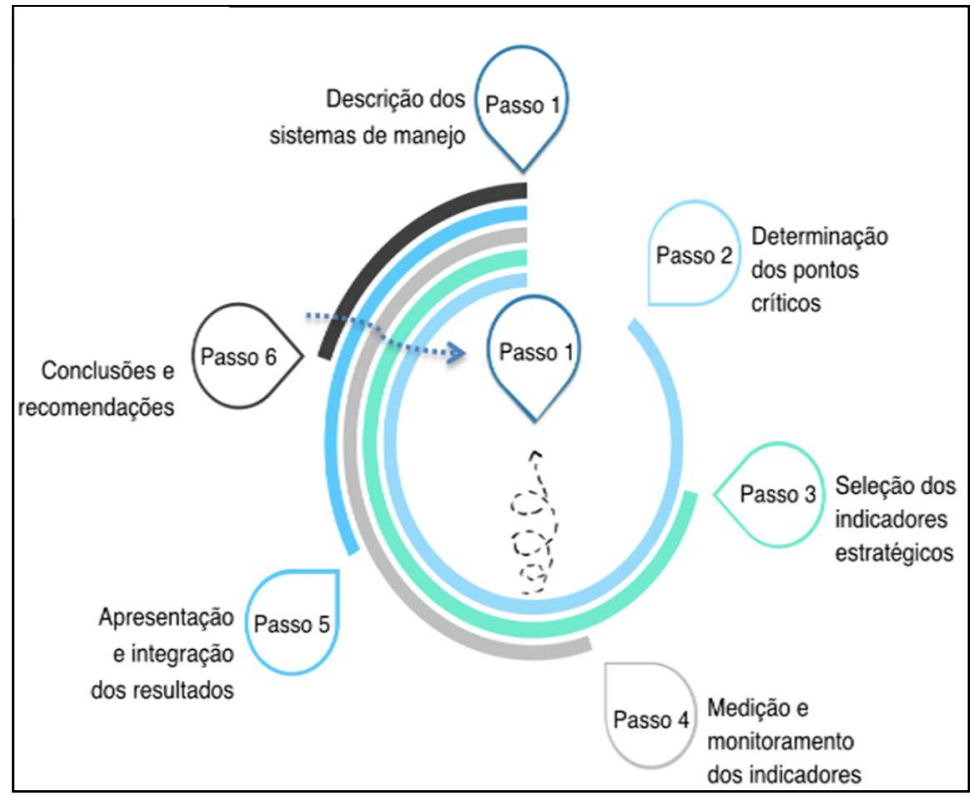

Figura 2: Ciclo de Avaliação do MESMIS. Fonte: Masera et al. (2000).

Assim sendo, o MESMIS pode avaliar uma propriedade individualmente tanto no modo transversal (comparando algumas propriedades durante o mesmo ciclo de produção) quanto no modo longitudinal, acompanhando os agroecossistemas no transcorrer do tempo (PEREIRA et al., 2010). A análise pelo método MESMIS possui vista na dimensão social, ambiental e econômica quanto a sua sustentabilidade e a sua aplicabilidade. Por conseguinte, a metodologia adotada para identificação dos pontos críticos proposto pelo MESMIS permite a avaliação da sustentabilidade em agroecossistemas de gestão familiar.

O uso dos passos de aplicação do método MESMIS em torno da amplitude das dimensões propostas, bem como da observação direta dos pesquisadores na busca de subsídios, permitem confrontar os dados, com a perspectiva de compreender com maior precisão a realidade dos agroecossistemas, pois eles apresentam como características comuns a agricultura familiar, base de produção alicerçada na agroecologia, foco na produção cultivo da manga vendida por atravessadores, além de produtos comercializados em feiras ou em outros meios alternativos locais.

De acordo com Masera et al. (2000), para dar solidez aos atributos gerais, definem-se uma série de pontos críticos e para cada área de avaliação se definem critérios de diagnóstico e indicadores. Assegurando uma relação clara entre os indicadores e os atributos da sustentabilidade do agroecossistema. A proposta metodológica para toda a avaliação de sustentabilidade deve estar ancorada na observação da agricultura sustentável, ou seja, em atributos, dimensões e indicadores de sustentabilidade, o que permite que sejam feitas propostas alternativas. 
A estrutura é construída sobre quatro princípios: os atributos do agroecossistema sustentável (produtividade, estabilidade, resiliência, confiabilidade, adaptabilidade, equidade e auto dependência) serão abordados por meio dos pontos críticos que, por sua vez, serão utilizados para determinação dos critérios de diagnóstico, os quais se direcionaram à seleção dos indicadores de sustentabilidade; as avaliações de sustentabilidade são válidas apenas para um sistema de gestão específico em uma escala espacial e temporal específica; o processo de avaliação é participativo para captar opiniões diversas; e a sustentabilidade é avaliada através da comparação de sistemas simultaneamente ou ao longo do tempo (MASERA et al., 2000).

Para verificar os pontos críticos, foram precisos determinar quais as situações fortalecem ou limitam a sustentabilidade do agroecossistema. Isso foram realizados com as observações dos procedimentos comuns dos agricultores em relação a seu agroecossistema e com diálogos com os agricultores dos agroecossistemas.

\section{RESULTADOS E DISCUSSÃO}

Os aspectos específicos dos processos aqui elencados estão apresentados em conformidade com as dimensões ambiental, econômica e social. A fim de alcançar o desenvolvimento sustentável, faz-se importante entender as relações entre tais dimensões e a forma de abordagem e desenvolvimento das relações entre sociedade e natureza (GALLOPÍN, 2006).

Nesse contexto, os critérios do diagnóstico descrevem os atributos da sustentabilidade e a interrelação entre atributo, pontos críticos e indicadores (MASERA et al., 2000; PEREIRA et al., 2010). A sustentabilidade em cada dimensão é, pois, definida como um conjunto de requisitos que devem ser satisfeitos por seus agroecossistemas, independentemente das diferenças na gestão da unidade de produção, do nível econômico e social da família, cobrindo, sempre, as três dimensões ou áreas de avaliação.

O método MESMIS possui a flexibilidade e possibilita a realização de adaptações técnicas no processo de avaliação e permite reconhecer as particularidades, buscando aproximar-se ao máximo da realidade de cada estudo (VERONA, 2010). Assim, foram determinados 7 (sete) pontos críticos: Solos, Recursos hídricos, Uso e ocupação do solo e sua conservação, Recursos financeiros, Formas de acesso à terra, Qualidade de vida e Organização e gestão, aspectos esses relacionados na dimensão ambiental, econômica e social.

\section{Dimensão ambiental}

Para Vázquez et al. (2018), a dimensão ambiental integra o trabalho sustentável com os elementos bióticos, abióticos e socioeconômicos com os quais o homem interage. Dessa forma, tal dimensão indica que ainda existe um interesse em um processo de manutenção da diversidade natural local, a qual é primordial para os agricultores da região. Nesse sentido, a ausência de uma prática de reciclagem do lixo é um ponto crítico, logo, é recomendável que o produtor transporte regularmente os resíduos sólidos de menor porte para pontos de coleta na zona urbana para que seja realizada a coleta seletiva pelo município. 


\section{Dimensão econômica}

Conforme pontuado por Vázquez et al. (2018), a dimensão econômica é condição essencial para o desenvolvimento, realizada a partir da identidade dos territórios e de seu potencial endógeno e produtivo. Desse modo, foi possível verificar que a renda econômica dos quatros agricultores são fatores de maior importância para a manutenção dos agroecossistemas rurais, o que torna a dimensão econômica predominante em aspectos regulares e ideais de sustentabilidade.

\section{Dimensão social}

Os indicadores sociais são orientados para avaliar a satisfação do produtor, sua qualidade de vida, sua integração social e seu conhecimento ecológico (SARANDÓN, 2002). Como pontua Sen (2000), as pessoas podem sofrer privações em diversas esferas da vida e seu bem-estar podem ser aferidos por meio das oportunidades sociais, liberdades políticas, facilidade econômica, bem como, das garantias de transparência, pois, as privações sofridas determinarão o posicionamento dos cidadãos em outras esferas. Com isso, percebe-se que a dimensão social possui foco na disponibilidade de serviços básicos e no fortalecimento das capacidades de recursos humanos, bem como salientam a importância da participação das instituições (públicas e privadas) e da sociedade organizada na construção de condições sociais aceitáveis, como serviços de saúde, escolaridade da família, cultura, lazer, entretenimento, entre outros.

\section{Procedimento dos passos pelo método MESMIS}

O procedimento estabelecido do ciclo de avaliação do MESMIS, sucedem da seguinte forma (MASERA et al., 2000): Passo 1: Descrição dos sistemas de manejo: Foram realizadas a caracterização a partir da observação dos procedimentos comuns dos agricultores em relação a seu agroecossistema de base familiar, com ênfase na descrição dos fatos sobre o cultivo da manga irrigada e da sequeiro com os quatro 'chefes' de família que residem no perímetro da comunidade rural de Acari e Cruzeta (RN).

Passo 2: Determinação dos pontos críticos: Nesse passo foram analisados os pontos críticos do sistema de agroecossistema, onde foram identificados através da relação aos elementos presentes na dimensão econômica, social e ambiental com o intuito de diagnosticar os pontos fracos e fortes relacionados à sustentabilidade. Após as visitas no local, com visita as 4 (quatro) famílias que cultivam o plantio de manga, seja irrigado, quanto de sequeiro, permitiu identificar uma série de limitações e potencialidades dessas unidades, da qual serviram quão grandemente pré-requisitos para a seleção dos indicadores do próximo passo. Segundo Verona (2008), indicador não é apenas uma informação tão somente numérica, ele deve descrever um processo específico ou de controle para cada sistema estudado, relacionado diretamente com a escala espacial em estudo.

Passo 3: Seleção dos critérios estratégicos: Nesse passo foram selecionados os indicadores para avaliar a sustentabilidade na comunidade rural do município de Acari e Cruzeta (RN). A lista de indicadores, foram baseadas desde a base teórica até a observação dos atores locais e levando em conta, o levantamento 
de dados primários de forma participativa de acordo com os atributos propostos pelo método MESMIS: produtividade, adaptabilidade, estabilidade, resiliência, confiabilidade, equidade e autogestão.

Para Masera et al. (2000), a sustentabilidade proposta por esse método se expressa em três dimensões: ecológica, econômica e sociocultural e elas se relacionam com cinco atributos gerais dos agroecossistemas. Os atributos básicos são essenciais ao estabelecer uma situação de agricultura sustentável que permite a derivação, medição e monitoramento de indicadores de sustentabilidade como parte de um processo flexível de avaliação participativa, sistêmica e interdisciplinar (MASERA et al., 2000).

Com essa sistematização, observar-se que os indicadores e parâmetros, foram construídos com o objetivo compor uma ferramenta útil para os seus principais usuários, como também obter informações sobre uma dada realidade a fim de se entender seus aspectos críticos. Assim, durante as visitas realizadas foi possível identificar os seguintes pontos, conforme a tabela 1.

Tabela 1: Potencialidades e limitações e indicadores elaborados.

\begin{tabular}{|c|c|c|}
\hline ATRIBUTOS & POTENCIALIDADES E LIMITAÇõES & DIMENSÃO \\
\hline \multirow{3}{*}{ Produtividade } & Média rentabilidade & Econômica \\
\cline { 2 - 4 } & Eficiência de utilização do trabalho familiar & Social \\
\cline { 2 - 4 } & Custo de manutenção é pouco variável & Econômica \\
\hline Estabilidade, & Inexistência de análises laboratoriais química, física e biológica do solo e água provenientes de poços & Ambiental \\
\hline & Presença do subsistema de criação animal & Ambiental \\
\hline Resiliência, & Deficiência da assistência técnica em relação ao manejo & Social \\
\hline & Áreas degradas & Ambiental \\
\hline & Canais permanentes de comercialização & Econômica \\
\hline Confiabilidade & Período muito longo de seca & Ambiental \\
\hline & Participação religiosas, de trabalho, associações & Social \\
\hline & Não utilização de insumos químicos e fertilizantes & Ambiental \\
\hline \multirow{2}{*}{ Adaptabilidade } & Resíduos sólidos (reciclagem) & Ambiental \\
\cline { 2 - 4 } & Aumento da eficiência do uso da água & Econômica \\
\hline \multirow{2}{*}{ Equidade } & Condições de habitação e saneamento & Social \\
\cline { 2 - 4 } & Acesso à saúde, educação, e programas governamentais & Social \\
\hline \multirow{2}{*}{ Autogestão } & Produção para autoconsumo & Econômica \\
\cline { 2 - 4 } & Acesso ao crédito rural & Econômica \\
\cline { 2 - 4 } & Falta de assistência técnica e extensão rural & Sociocultural \\
\cline { 2 - 4 } & Aquisição de produtos agropecuários & Ambiental \\
\hline
\end{tabular}

Assim, pode-se eleger 19 indicadores, partindo dos estudos desenvolvidos por Lora (2019), com agricultores participantes da feira do produtor em Pato Branco (PR) e Gallo et al. (2016), em uma propriedade rural de base familiar, situado em Vila Bella, localizada no município de Glória de Dourados, Mato Grosso do Sul, onde foram avaliadas em ambos trabalhos a sustentabilidade de unidade de produção agrícola, tendo como base três parâmetros propostos pelo método (MASERA et al., 2000), onde atribui a importância aos aspectos econômicos da sustentabilidade, avaliando os parâmetros como o valor presente líquido, a relação custo $\mathrm{x}$ benefício e lucratividade.

Passo 4: Medição e monitoramento dos indicadores: Neste passo, é utilizado a mensuração dos indicadores estratégicos através da formulação de instrumentos de avaliação, com o objetivo de transformar as variáveis qualitativas em quantitativa. A observação direta, possibilitou coletar dados, com perguntas relacionadas aos indicadores, da qual permitiam responder aos indicadores estabelecidos no estágio anterior (NICOLOSO et al., 2018).

Seguindo o detalhamento e avaliação do método na construção dos indicadores proposto por Verona (2008) e utilizado por Lora (2019), tendo em consideração o somatório dos parâmetros processados em cada 
indicador no que concerne ao grau de sustentabilidade do local de estudo, foram atribuídos notas de parâmetros para cada indicador avaliado, sendo de 1 (um) a 3 (três), onde, 1 (um) apresentam uma condição não desejável, 2 (dois) representam uma condição regular e 3 (três) representam condições desejáveis, de acordo com as premissas para a sustentabilidade (tabela 2).

De acordo com Sperrhake et al. (2012), a partir da década de 1950, uma exigência da Unesco, as pesquisas domiciliares realizados pelo IBGE referente aos não alfabetizados, mudou, passando a ser considerada analfabeta a pessoa que declara não saber ler e escrever um bilhete simples no idioma que conhece.

Tabela 2: Indicadores e parâmetros.

\begin{tabular}{|c|c|c|c|c|c|}
\hline \multirow{2}{*}{ DIMENSÕES } & \multirow{2}{*}{ № } & \multirow{2}{*}{ INDICADORES } & \multicolumn{3}{|c|}{ PARÂMETROS } \\
\hline & & & 1 & 2 & 3 \\
\hline \multirow{4}{*}{ 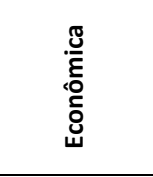 } & 1 & Renda Econômica & Salário mínimo & 2 a 3 salários & Acima de 3 salários \\
\hline & 2 & Produção Agrícola & Pouca & Razoável & Acima da média \\
\hline & 3 & Implementos Agrícolas & Modo intensivo & Manual & Quando necessário \\
\hline & 4 & $\begin{array}{l}\text { Comercialização da } \\
\text { produção }\end{array}$ & Com intermediário & $\begin{array}{l}\text { Intermediário + venda } \\
\text { direta }\end{array}$ & $\begin{array}{l}\text { Venda direta (feiras, local de } \\
\text { produção, etc.) }\end{array}$ \\
\hline \multirow{11}{*}{ 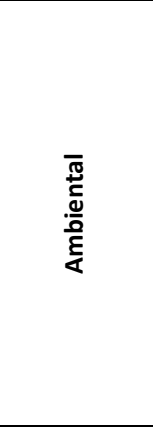 } & 5 & Uso de recursos naturais & Não faz & Faz, sem manejo & Faz, com manejo \\
\hline & 6 & $\begin{array}{c}\text { Água para consumo } \\
\text { humano }\end{array}$ & Não tratada & Filtrada & Faz, com manejo \\
\hline & 7 & Água para agricultura & Não tratada & Filtrada & Tratada \\
\hline & 8 & Esgoto & Ambiente & Fossa & Tradada \\
\hline & 9 & Reciclagem do lixo & Não faz & Faz parcialmente & Faz $100 \%$ \\
\hline & 10 & Cobertura do solo & Solo exposto & Com cultivos & Cobertura em todo o ano \\
\hline & 11 & Adubação & $50 \%$ orgânico & $<90>50 \%$ orgânico & $>90 \%$ orgânico \\
\hline & 12 & Áreas degradadas & Várias & Poucas & Não há \\
\hline & 13 & Desmatamento & Já realizou & Parcialmente & Nunca houve \\
\hline & 14 & Queimadas & Já realizou & Parcialmente & Nunca houve \\
\hline & 15 & Análise e correção do solo & Não faz & Faz esporadicamente & Sempre que necessário \\
\hline \multirow{4}{*}{. } & 16 & Atuação de cooperativas & Não tem & Existe parcialmente & Existe integralmente \\
\hline & 17 & Mão de obra terceirizada & $\begin{array}{l}\text { Para todas as } \\
\text { atividades }\end{array}$ & Apenas algumas & Não há \\
\hline & 18 & $\begin{array}{l}\text { Ajuda de programas } \\
\text { sociais }\end{array}$ & Não tem & Recebe pouco & Recebe significativamente \\
\hline & 19 & Escolaridade & Não alfabetizados & Alfabetizados & $\begin{array}{l}\text { Alfabetizados com segundo grau } \\
\text { completo }\end{array}$ \\
\hline
\end{tabular}

Ao levar em conta os atributos identificados, o MESMIS propõe que, para estabelecer uma definição operativa do conceito de sustentabilidade, é necessário identificar uma série de propriedades ou atributos gerais de agroecossistemas sustentáveis (MASERA et al., 2000). Pode-se identificar as potencialidades e limitações do sistema de gestão relacionadas aos diferentes atributos que determinam a sustentabilidade.

Dentre eles, podemos citar: a necessidade de contornar os problemas da falta de água; a maneira de rentabilizar a produtividade de forma eficiente a partir da utilização de menos recursos pela falta da assistência técnica; a deficiência no autogerenciamento por parte dos agricultores em relação à sua produtividade e comercialização; o acesso ao crédito para a competitividade dos pequenos negócios da fruticultura; e o saneamento e moradia, os quais são essenciais no estabelecimento de medidas de promoção da qualidade de vida do agricultor familiar.

Passo 5: Apresentação e integração dos resultados: Neste passo, poderá ser analisados os resultados apresentados pelos agroecossistemas pesquisado, onde as avaliações quantitativas e qualitativas são transcorridas para valores numéricos. Segundo Verona (2008), uma característica do método MESMIS 
consiste na exigência da abordagem das dimensões ambientais e socioeconômicas, onde os valores de referência são estabelecidos para cada indicador.

Para Souza et al. (2017), nesta fase deve ser feita a apresentação dos resultados obtidos com o objetivo de auxiliar na tomada de decisões sobre os caminhos a serem traçados para a melhoria dos sistemas, buscando uma forma possível de mostrar os resultados encontrados. Por essa razão, é preciso buscar dentro do MESMIS o procedimento para mostrar os resultados, onde fiquem claro os problemas dos agroecossistemas para que seus indicadores sejam agrupados dentro dos três pilares da sustentabilidade, ambiental, econômico e social.

Passo 6: Conclusões e recomendações: Conforme Souza et al. (2017), esse último passo tem como objetivo a apresentação de conclusões claras sobre sustentabilidade do sistema pesquisado e recomendações para melhorar a sustentabilidade dos mesmos. Neste passo poderá ser apresentados os resultados das análises, conclusões e recomendações para a sustentabilidade dos agroecossistema e finalizar o ciclo. Ao realizar todo o ciclo do MESMIS, poderá ser entendido o agroecossistema, os aspectos que deseja melhorar, além de poder indicar uma série de recomendações que dará início a um redesenho do agroecossistema, permitindo assim, uma proposta de monitoramento e acompanhamento constantes à saber qual dos 2 (dois) tipos de cultivo é mais sustentável.

\section{CONSIDERAÇÕES FINAIS}

É importante destacar que a aplicação do método MESMIS nos agroecossistemas pesquisados se deu por meio do entendimento de agricultura sustentável, nas pesquisas teóricas e nas análises dos dados primários coletados no campo pelo pesquisar, bem como, os dados secundários. Esta análise evidencia que a aplicação do método MESMIS nos agroecossistemas de base familiar do cultivo da manga irrigada e de sequeiro, a partir de indicadores de sustentabilidade, contribui tanto com o conhecimento sobre o modo de funcionamento do agroecossistema quanto com a caracterização da gestão da unidade de produção familiar. Nesse sentido, esse método, com sua proposta de abordagem participativa, de estrutura flexível e adaptável, mostra-se mais adequado à avaliação destas unidades produtivas agroecológicas na Agricultura Familiar.

Segundo Masera et al. (2000), demora vários anos para obter resultados confiáveis (5-10 anos), até você ver uma tendência clara. Vale ressaltar que, embora este método seja bastante preciso, baseia seu sucesso na escolha dos indicadores corretos e em seu ciclo completo. Após a realização desses seis passos tornará possível obter um melhor entendimento do agroecossistema em estudo, como também poderá ser observado o que será necessário ser aprimorado nele.

A análise conduzida neste artigo demonstra, também, que as abordagens da cultura de manga irrigada e de sequeiro possuem vantagens e desvantagens particulares. Além disso, a pesquisa mostrou que foi possível identificar até o passo 4 (quatro) obter, quantificar as informações desejadas, a fim de que, a construção de ferramentas práticas possa ser inserida na agricultura familiar para concretizar o processo de uma agricultura sustentável. Portanto, é possível afirmar que os objetivos propostos na pesquisa, ou seja, os de apontar os aspectos gerais da utilização de indicadores como instrumento de avaliação da 
sustentabilidade, determinando os pontos críticos e definindo seus parâmetros, foram plenamente atingidos.

\section{REFERÊNCIAS}

BRASIL. Lei n.11326 de 24 de julho de 2006. Estabelece as diretrizes para a formulação da Política Nacional da Agricultura Familiar e Empreendimentos Familiares Rurais. Brasília: DOU, 2006.

BRASIL. Ministério da Agricultura, Pecuária e Abastecimento. Características da planta. Brasília: MAPA, 2007.

BRASIL. Ministério da Agricultura, Pecuária e Abastecimento. Estímulo à produção autônoma e sustentável dos agricultores familiares. Brasília: MAPA, 2018.

BRASIL. Ministério de Minas e Energia. Projeto cadastro de fontes de abastecimento por água subterrânea: Diagnóstico do município de Acari e Cruzeta, estado do Rio Grande do Norte. Brasília: MME, 2005.

BRASIL. Ministério do Desenvolvimento Agrário. Agricultura familiar. Brasília: MDA, 2016.

BRASIL. Ministério do Desenvolvimento Social. Estratégia Intersetorial de prevenção e controle da obesidade: recomendações para estados e municípios. Brasília: MDS, 2014.

BROSE, M.. Agricultura familiar: desenvolvimento local e políticas públicas. Santa Cruz do Sul: EDUNISC, 1999.

BUAINAIN, A. M.. Agricultura familiar, agroecológica e desenvolvimento sustentável: questões para debate. Brasília: IICA, 2006.

CAMELO, G. P.; CÂNDIDO, G. A.. Potencialidades e Limitações dos Agroecossistemas Familiares de Cultivo do Abacaxi em Touros (RN). Holos, v.6, p.3-27, 2013. DOI: https://doi.org/10.15628/holos.2012.1124

CÂNDIDO, G. A.; NÓBREGA, M. M.; FIGUEIREDO, M. T. M.; MAIOR, M. M. S.. Avaliação da Sustentabilidade de Unidades de Produção Agroecológicas: Um Estudo Comparativo dos Métodos IDEA e MESMIS. Ambiente \& Sociedade, São Paulo, v.18, n.3, p.99-120, 2015. DOI: http://doi.org/10.1590/1809-4422ASOC756V1832015

CÂNDIDO, G. A.; SILVA, V. P.. Indicadores de sustentabilidade em agroecossistemas: aplicações em diversos tipos de cultivo e práticas agrícolas do Rio Grande do Norte. Natal: EDIFRN, 2015.

CASTRO, N.. Desafios da agricultura familiar: o caso da assistência técnica e extensão rural. Boletim Regional, Urbano e Ambiental, n.12, 2015.

COSTA, A. N.; COSTA, A. F. S.; CAETANO, L. C. S.; VENTURA, J. A.. Recomendações técnicas para a produção de manga. Vitória: Incaper, 2008.

CUNHA, W. A.; FREITAS, A. F.; SALGADO, R. J. S. F. Efeitos dos Programas Governamentais de Aquisição de Alimentos para a Agricultura Familiar em Espera Feliz, MG. Revista de
Economia e Sociologia Rural, Brasília, v.55, n.3, p.427-444, 2017. DOI: http://doi.org/10.1590/1234-56781806$\underline{94790550301}$

DEIMLING, M. F.; BARICHELLO, R.; BRAZ, R. J.; BIEGER, B. N.; CASAROTTO FILHO, N.. Agricultura familiar e as relações na comercialização da produção. Interciencia, v.40, n.7, 2015.

DELGADO, G. C; BERGAMASCO, S. M. P. P.. Agricultura Familiar Brasileira: Desafios e Perspectivas de Futuro. Brasília: MDA, 2017.

FERRAZ, J. M. G.; PESSOA, M. C. P. Y.; MARQUES, J. F.; SKORUPA, L. A.. Construção participativa de indicadores de sustentabilidade. Jaguariúna: 2004.

FORMIGA JÚNIOR, I. M. F.; CÂNDIDO, G. A.; AMARAL, V. S.. Sustentabilidade do cultivo de melão no Assentamento São Romão em Mossoró/RN: determinação dos pontos críticos. Campo-Território: Revista de Geografia Agrária, v.9, n.19, p.57-87, 2014.

GALLO, A. S.; GUIMARÃES, N. F.; CUNHA, C.; SANTOS, R. D.; CARVALHO, E. M.. Indicadores da sustentabilidade de uma propriedade rural de base familiar no estado de Mato Grosso do Sul. Revista Verde de Agroecologia e Desenvolvimento Sustentável, Pombal, v.11, n.3, p.104-114, 2016.

GALLOPÍN, G. C.. Environmental and sustainability indicators and the concept of situational indicators: A systems approach. Environmental Modeling \& Assessment, v.1, n.3, p.101-117, 1996

GALLOPÍN, G. C.. Los indicadores de desarrollo sostenible: aspectos conceptuales y metodológicos. Santiago: 2006.

GERHARDT, T. E.; SILVEIRA, D. T.. Métodos de pesquisa. Porto Alegre: EDUFRGS, 2009.

GIL, A. C.. Como Elaborar Projetos de Pesquisa. 5 ed. São Paulo: Atlas. 2010

GLIESSMAN, S. R.. Agroecologia: processos ecológicos em agricultura sustentável. 2 ed. Porto Alegre: EDUFRGS, 2009.

GONSALVES, E. P.. Conversas sobre iniciação à pesquisa científica. Campinas: Alínea, 2001.

IBGE. Instituto Brasileiro de Geografia e Estatística. Censo Agropecuário 2017. Rio de Janeiro: IBGE, 2017.

LAKATOS, E. M.; MARCONI, M. A.. Técnicas de pesquisa. 6 ed. São Paulo: Atlas, 2007.

LORA, M. I.. Avaliação da sustentabilidade de agroecossistemas dos agricultores participantes da feira do produtor em Pato Branco-PR: uma abordagem ao longo do tempo. Dissertação (Mestrado em Desenvolvimento 
Regional) - Universidade Tecnológica Federal do Paraná, Pato Branco, 2019.

MARTINS, J. P. S.. A contribuição da agricultura para os Objetivos de Desenvolvimento Sustentável. São Paulo: ASN, 2018.

MASCARENHA, G. C. C.. A atual conjuntura socioeconômica e ambiental da região Sul da Bahia e a agricultura sustentável como uma alternativa concreta. In: UZÊDA, M. C.. O desafio da agricultura sustentável: alternativas viáveis para o Sul da Bahia. Ilhéus: Editus, 2004. p.13-32.

MASERA, O.; ASTIER, M.; LÓPEZ-RIDAURA, S.. Sustentabilidad y manejo de recursos naturales: el marco de evaluación MESMIS. Cidade do México: 2000.

NICOLOSO, C. S.; SILVEIRA, V. C. P.; COELHO FILHO, R. C.; QUADROS, F. L. F.. Aplicação do Método MESMIS para Análise da Sustentabilidade de Sistemas de Produção da Pecuária Familiar em Área do Bioma Pampa no Rio Grande do Sul. Desenvolvimento em Questão, n.45, p.354-376, 2018. DOI: http://doi.org/10.21527/2237-6453.2018.45.35437

OLIVEIRA, M. L. R.. Reflexões sobre o uso de metodologias participativas como instrumento de trabalho em comunidades rurais. Em Extensão, v.14, n.1, p.30-51, 2015. DOI: https://doi.org/10.14393/REE-v14n12015 art02

PEREIRA, V. S.; MARTINS, S. R.. Indicadores de sustentabilidade do agroecossistema arroz orgânico com manejo de água contínuo na bacia do Araguá (SC) mediante aplicação da metodologia MESMIS. Revista Brasileira de Ciências Ambientais, v.15, n.1, p.56-78, 2010.

PETERSEN, P.; SILVEIRA, L. M.; FERNANDES, G. B.; ALMEIDA, S. G.. Método de Análise Econômico-Ecológica de agroecossistemas: Articulação Nacional de Agroecologia (Brasil). Rio de Janeiro: AS-PTA, 2017.

QUARANTA, G.. Land Care in Desertification Affected Areas: Project Lucinda. Lisboa: ICNF, 2000.

SANTOS, C. F.; SIQUEIRA, E. S.; ARAUJO, I. T.; MAIA, Z. M. G.. $A$ agroecologia como perspectiva de sustentabilidade na agricultura familiar. Ambiente \& Sociedade, São Paulo, v.17, n.2, p.33-52, 2014. DOI: http://doi.org/10.1590/S1414$\underline{753 \times 2014000200004}$

SARANDÓN, S. J.. La agricultura como actividad transformadora del ambiente: El Impacto de la Agricultura intensiva de la Revolución Verde. Ediciones Científicas Americanas, La Plata, p.393-414. 2002.

SCHNEIDER, S.; MATTEI, L.; CAZELLA, A. A.. Histórico, caracterização e dinâmica recente do Pronaf: Programa Nacional de Fortalecimento da Agricultura Familiar. In: SCHNEIDER, S.; SILVA, M. K.; MARQUES, P. E.. Políticas Públicas e Participação Social no Brasil Rural. Porto Alegre: UFRGS, 2004.

SEN, A. K.. Desenvolvimento como Liberdade. São Paulo: Companhia das Letras, 2000.

SILVA, C. R. R.; FONSECA, E. B. A.; MOREIRA, M. A.. A cultura da mangueira. Minas Gerais: EDUFLA, 2002.

SILVA, E. F.; REIS, L. M.. Situação ambiental de uma comunidade rural no semiárido potiguar. Geolnterações, Assú, v.1, n.1, p.69-85, 2017.

SOUZA, R. T. M.; MARTINS, S. R.; VERONA, L. A. F.. A metodologia MESMIS como instrumento de gestão ambiental em agroecossistemas no contexto da Rede CONSAGRO. Agricultura Familiar, v.11, n.1, p.39-56, 2017.

SPERRHAKE, R.; TRAVERSINI, C. S.. Os critérios do Censo produzindo estatísticas de alfabetização: gerenciar o risco e inventar pessoas. Estatística e Sociedade, Porto Alegre, n.2, p.142-156, 2012.

VARGAS, D. L.; HILLIG, C.; NETTO, T. A.. A necessidade de agroecossistemas sustentáveis frente ao cenário social e ambiental na atualidade. Monografias Ambientais, v11, n.10 p.2260-2269, 2012.

VÁZQUEZ, M. M.; YAINER, M. M.. Evaluación de las dimensiones económica y socioambiental en un agroecosistema del municipio Yara. Revista Caribeña de Ciencias Sociales, Bayamo, 2018.

VERONA, L. A. F.. A real sustentabilidade dos modelos de produção da agricultura: Indicadores de sustentabilidade na agricultura. Revista da Associação Brasileira de Horticultura, v.28, n.2, 2010.

VERONA, L. A. F.. Avaliação de sustentabilidade em agroecossistemas de base familiar e em transição agroecológica na região sul do Rio Grande do Sul. Tese (Doutorado em Agronomia) - Universidade Federal de Pelotas, Pelotas, 2008.

A CBPC - Companhia Brasileira de Produção Científica (CNPJ: 11.221.422/0001-03) detém os direitos materiais desta publicação. Os direitos referem-se à publicação do trabalho em qualquer parte do mundo, incluindo os direitos às renovações, expansões e disseminações da contribuição, bem como outros direitos subsidiários. Todos os trabalhos publicados eletronicamente poderão posteriormente ser publicados em coletâneas impressas sob coordenação da Sustenere Publishing, da Companhia Brasileira de Produção Científica e seus parceiros autorizados. Os (as) autores (as) preservam os direitos autorais, mas não têm permissão para a publicação da contribuição em outro meio, impresso ou digital, em português ou em tradução. 